\title{
Patterns of access to reproductive health services in Ghana and Nigeria: results of a cluster analysis
}

\author{
Oluwasegun Jko Ogundele ${ }^{1 *}$, Milena Pavlova ${ }^{1}$ and Wim Groot ${ }^{1,2}$
}

\begin{abstract}
Background: Inequalities in access to health care result in systematic health differences between social groups. Interventions to improve health do not always consider these inequalities. To examine access to reproductive health care services in Ghana and Nigeria, the patterns of use of family planning and maternal care by women in these countries are explored.
\end{abstract}

Methods: We used population-level data from the Ghana and Nigeria Demographic Health Surveys of 2014 and 2013 respectively. We applied a two-step cluster analysis followed by multinomial logistic regression analysis.

Results: The initial two-step cluster analyses related to family planning identified three clusters of women in Ghana and Nigeria: women with high, medium and poor access to family planning services. The subsequent two-step cluster analyses related to maternal care identified five distinct clusters: higher, high, medium, low and poor access to maternal health services in Ghana and Nigeria. Multinomial logistic regression showed that compared to women with secondary/higher education, women without education have higher odds of poor access to family planning services in Nigeria ( $\mathrm{OR}=2.54,95 \% \mathrm{Cl}: 1.90-3.39)$ and in Ghana ( $\mathrm{OR}=1.257,95 \% \mathrm{Cl}$ : 0.77-2.03). Compared to whitecollar workers, women who are not working have increased odds of poor access to maternal health services in Nigeria ( $\mathrm{OR}=1.579,95 \% \mathrm{Cl}: 1.081-2.307, p \leq 0.01)$. This association is not observed for Ghana. Household wealth is strongly associated with access to family planning services and maternal health care services in Nigeria. Not having insurance in Ghana is associated with low access to family planning services, while this is not the case in Nigeria. In both countries, the absence of insurance is associated with poor access to maternal health services.

Conclusions: These differences confirm the importance of a focused context-specific approach towards reproductive health services, particularly to reduce inequality in access resulting from socio-economic status. Interventions should be focused on the categorization of services and population groups into priority classes based on needs assessment. In this way, they can help expand coverage of quality services bottom up to improve access among these vulnerable groups.

Keywords: Reproductive services, Maternal care, Family planning, Access, Nigeria, Ghana

\footnotetext{
* Correspondence: j.ogundele@maastrichtuniversity.nl

'Department of Health Services Research; CAPHRI, Maastricht University Medical Center; Faculty of Health, Medicine and Life Sciences; Maastricht University, PO Box 616, 6200MD, Maastricht, The Netherlands

Full list of author information is available at the end of the article
} 


\section{Background}

Inequalities in access to health care can result in health differences between social groups. Interventions to create universal access to health care and to improve health outcomes, do not always consider these inequalities. Women are exposed to unequal access to health care services globally [1]. This is particularly the case for reproductive health care services [2], which include contraceptives, maternal health services, and services related to sexual health [3-5]. Targets have been set to improve these services. For example, the Sustainable Development Goal 3 aims to ensure universal access to reproductive health care services [6].

Factors that determine access to reproductive health services are related to both demand and supply and can be divided into social and economic factors [7]. Education, occupation, wealth and possession of insurance, among others, are significant predictors of inequality in access to reproductive health services in Sub-Saharan Africa [8-13]. However, previous studies have mostly focused on the determinants of service use in a country or region $[11,14,15]$. There is a need for cross-country comparisons to shed light on similarities and/or dissimilarities between groups of users of reproductive health services in Sub-Saharan African countries.

This study examines access to reproductive health care services among women of reproductive age in Ghana and Nigeria. We use data from the Demographic Health Surveys (DHS) of Ghana carried out in 2014 and that of Nigeria carried out in 2013. The two countries are selected for this study based on the similarities in trends, health outcomes as well as data availability. At the same time, their health care systems are different. For example, Ghana has an established national health insurance system, while there is no such well-established system in Nigeria [16]. Ghana and Nigeria introduced a minimal user fee in the early 1970s, which was later abandoned in both countries due to cash crunches [17]. Ghana offers free-of-charge maternal care. The health insurance scheme in the country is reported to cover $65 \%$ of the population, which reduces the out-of-pocket health expenditure (66\% of total health spending) [18]. One study using a cluster analysis method has shown that there are differences in the adequacy of maternal care available in Ghana and that there are disparities in the socio-demographic characteristics that determine access [8]. Insurance in Nigeria covers 3.5\% of the population with out-of-pocket health expenditure amounting to over $90 \%$ of total health spending $[17,18]$. These differences are expected to result in differences in access to reproductive health services, which we investigate in this paper.

\section{Methods}

The DHS are nationally representative cross-sectional surveys carried out in low- and middle-income countries periodically $[19,20]$. The DHS adopts a multi-stage sampling design. Samples selected for enumeration, are ensured to be representative and comparative across countries. The DHS of Ghana and Nigeria included in this study, involved a two-stage sampling procedure: first selecting the location and then, selecting households per location at random [19, 20]. Within a household, respondents were selected by gender for the different questionnaire types. A respondent was included if he/ she was a usual member of the household or had spent the night preceding the survey, in the household.

We only used data for women of reproductive age (15-49 years) in Ghana and Nigeria who had given birth during the last 5 years before the survey and were able to provide information on the use of reproductive health services. Our study included 4142 women from the DHS of Ghana and 7725 women from the DHS of Nigeria. The indicators of reproductive care that we used in the cluster analysis are categorized as family planning services and maternal health services and are shown in Appendix A of the supplementary file.

We first performed two-step cluster analyses, which provided insight into the patterns of reproductive health services use among women of reproductive age in both countries. Cluster analysis is a method to classify similarities or dissimilarities based on respondents' data [21]. Four cluster analyses were carried out, namely one for family planning services and another one for maternal health services for each of the two countries. The clusters generated by the cluster analysis procedure are shown in Appendix A in the supplementary file. In particular, the two-step clustering procedure uses the Schwarz's Bayesian Information Criterion (BIC) method to determine the number of clusters. Different clustering solutions are compared and the clustering solution with the lowest BIC is selected by the procedure. We inspected this clustering and accept it as adequate. The stability and reliability of the cluster analyses were confirmed by repeating the clustering procedure produced no less than 10 times. The repeated analyses resulted in the same cluster quality. The two-step cluster analysis procedure specifies the clustering quality based on the Silhouette Index (SI). The SI indicates how well each subject/object lies within its cluster, and thus, it validates the clustering outcomes. The SI ranges from -1 to 1 . SI greater or equal to 0.5 indicates good clustering quality.

We titled the clusters based on the quality and adequacy of medical care used by women in each cluster compared to what is usually provided in governmentlicensed medical facilities. Thus, in the poor access cluster, on average, women reported using less and lower quality care than the care usually provided at government-licensed facilities, and in the high access cluster, women reported using more and better care. 
Details about the cluster composition variables, patterns, and quality are presented in Appendix A in the supplementary file.

Multinomial logistic regression was used to identify factors associated with the cluster membership determined during the cluster analyses. A total of four regression analyses were conducted. The cluster membership generated in each cluster analysis was the dependent variable in the multinomial logistic regression analyses. The explanatory variables consisted of women's background characteristics that were found to be associated with the use of family planning services and maternal health services in previous studies and were available in our dataset. Sample weights were applied for the multinomial logistic regression. Software package SPSS version 23 was used for all data analyses.

\section{Results}

Descriptive statistics on the socio-economic characteristics of the two samples and primary results of the twosteps cluster analyses can be found in the appendices (supplementary files). Below, we present the key findings of the cluster analyses as well as the results of the regression analyses.

\section{Cluster analysis}

The two-step cluster analysis of family planning service use in Ghana automatically produced 3 distinct clusters. In the two-step cluster analysis of family planning service use in Nigeria, the number of clusters (3 clusters) was fixed in advance to be able to produce meaningful clusters. The clusters are presented in Table 1 . The clusters were inspected and labeled as high, medium, and poor access to family planning services based on the

Table 1 Frequency distribution of cluster membership

\begin{tabular}{lllll}
\hline & \multicolumn{3}{l}{ Family planning services } \\
\cline { 2 - 5 } & Ghana & & Nigeria & \\
\hline Cluster group & Obs & $\%$ & Obs & $\%$ \\
Poor-access & 2755 & 64.2 & 5638 & 71.5 \\
Medium Access & 918 & 14.4 & 1507 & 9.3 \\
High-Access & 619 & 21.4 & 736 & 19.1
\end{tabular}

Maternal health service

\begin{tabular}{lllll} 
& Ghana & \multicolumn{3}{c}{ Nigeria } \\
& Obs & $\%$ & Obs & $\%$ \\
Low-access & 293 & 7.1 & 346 & 4.4 \\
Poor-Access & 1053 & 25.4 & 1452 & 18.5 \\
Medium-Access & 756 & 18.2 & 2027 & 25.9 \\
High-Access & 952 & 23.0 & 1693 & 21.6 \\
Higher-access & 1092 & 26.3 & 2315 & 29.6 \\
\hline
\end{tabular}

Note: The total percentage of Family planning in Nigeria does not add up to $100 \%$ due to approximation services used by women in each cluster (see Methods section). The cluster with high access to family planning services captures 19.1 and $21.4 \%$ of women in Nigeria's and Ghana's sample respectively. The other extreme is the third cluster that consists of women whose access can be described as poor; $71.5 \%$ of women in Nigeria's sample belong to this cluster and $64.2 \%$ of women in Ghana's sample.

We did not predefine the number of clusters for maternal health services. For both countries, the two-step cluster analyses of maternal health services use resulted in five clusters, which we inspected and labeled as higher, high, medium, low and poor access to maternal health services (see Table 1). The higher-access cluster captures $29.6 \%$ of women in Nigeria's sample and $26.3 \%$ of the women in Ghana's sample. Relative to the other four clusters, a larger proportion of members of this cluster report that they accessed government hospitals for antenatal care and used institutional maternal care more. The high-access cluster consists of $21.6 \%$ of women in Nigeria's sample and $23.0 \%$ of women in Ghana's sample. For both countries, this cluster has a lower proportion of women who accessed government health centers for antenatal care or got assistance from physicians during childbirth. Members of the medium-access cluster in both countries used private facilities for antenatal care as well as for childbirth. This cluster of women makes up $25.9 \%$ of Nigeria's sample and $18.2 \%$ of the Ghana sample. Members of the low-access cluster in both countries mostly report that they accessed government health posts/dispensaries for antenatal care but did not have skilled assistance during childbirth. In Nigeria's sample, $4.4 \%$ of women fall into this cluster and in the Ghana's sample, this share is $7.1 \%$. Lastly, 18.5 and $25.4 \%$ of women from the Nigeria's and Ghana's sample respectively are members of the pooraccess cluster. Members of this cluster mostly did not receive institutionalized maternal care. For both countries, the poor-access cluster has a high proportion of members who had home childbirth and used traditional birth attendants during childbirth.

\section{Regression analysis}

The dependent variables in the four multinomial logistic regressions were the four cluster membership variables generated in the cluster analyses. Tables 2 and 3 present the odds ratios for the four regressions. Information about the independent variables used and the full results of the regression analyses can be found in Appendix B of the supplementary file.

For family planning services, the results in Table 2 show that in both countries, women with no education, compared to women with secondary or higher education, have higher odds to belong to the poor-access 
Table 2 Odds ratio of use of family planning services in Nigeria and Ghana (multinomial logistic regression)

\begin{tabular}{|c|c|c|c|c|}
\hline \multirow[t]{4}{*}{ Background characteristics } & \multicolumn{2}{|l|}{ Nigeria } & \multicolumn{2}{|l|}{ Ghana } \\
\hline & Medium-access & Poor-access & Medium-access & Poor-access \\
\hline & \multicolumn{2}{|c|}{ Reference category: High access } & \multicolumn{2}{|c|}{ Reference category: High access } \\
\hline & $\operatorname{Exp} B(95 \% \mathrm{Cl})$ & Exp B $(95 \% \mathrm{Cl})$ & Exp B $(95 \% \mathrm{Cl})$ & $\operatorname{Exp} B(95 \% \mathrm{Cl})$ \\
\hline Maternal age & $1.018^{\mathrm{a}}(0.998-1.038)$ & $1.015^{\mathrm{b}}(1.001-1.029)$ & $1.047^{c}(1.023-1.072)$ & $1.037^{c}(1.019-1.055)$ \\
\hline Number of children alive & $0.940^{\mathrm{a}}(0.878-1.007)$ & $0.774^{c}(0.738-0.813)$ & $0.851^{c}(0.777-0.931)$ & $0.849^{\mathrm{C}}(0.795-0.908)$ \\
\hline \multicolumn{5}{|l|}{ Marital status } \\
\hline Married (ref) & 1 & 1 & 1 & 1 \\
\hline Never married & $0.338^{\mathrm{C}}(0.185-0.619)$ & $0.550^{c}(0.395-0.767)$ & $1.068(0.705-1.617)$ & $1.359^{\mathrm{b}}(1.008-1.833)$ \\
\hline Widowed/separated/divorced & $1.038(0.606-1.777)$ & $1.531^{\mathrm{b}}(1.052-2.228)$ & $1.004(0.656-1.535)$ & $0.935(0.695-1.258)$ \\
\hline \multicolumn{5}{|l|}{ Maternal Education } \\
\hline Secondary/ Higher (ref) & 1 & 1 & 1 & 1 \\
\hline No education & $1.257(0.776-2.037)$ & $2.544^{c}(1.907-3.395)$ & $1.350(0.941-1.938)$ & $1.527^{\mathrm{C}}(1.173-1.988)$ \\
\hline Primary & $0.825(0.642-1.061)$ & $1.111(0.939-1.314)$ & $0.817(0.601-1.112)$ & $0.961(0.774-1.192)$ \\
\hline \multicolumn{5}{|l|}{ Maternal Occupation } \\
\hline White collar (ref) & 1 & 1 & 1 & 1 \\
\hline Not working & $1.006(0.706-1.435)$ & $1.135(0.882-1.459)$ & $1.732^{\mathrm{b}}(1.027-2.921)$ & $2.194^{\mathrm{C}}(1.447-3.325)$ \\
\hline Services and manual & $1.134(0.806-1.594)$ & $1.283^{b}(1.002-1.642)$ & $1.227(0.723-2.081)$ & $1.727^{\complement}(1.137-2.622)$ \\
\hline Sales & $0.930(0.687-1.258)$ & $0.987(0.793-1.228)$ & $1.238(0.771-1.987)$ & $1.686^{\mathrm{C}}(1.152-2.466)$ \\
\hline Agriculture & $0.805(0.503-1.291)$ & $1.194(0.867-1.646)$ & $1.378(0.785-2.421)$ & $1.801^{c}(1.164-2.786)$ \\
\hline \multicolumn{5}{|l|}{ Household Wealth } \\
\hline Richest (ref) & 1 & 1 & 1 & 1 \\
\hline Poorest & $0.762(0.220-2.633)$ & $3.417^{c}(1.825-6.396)$ & $0.947(0.519-1.731)$ & $1.403(0.895-2.200)$ \\
\hline Poorer & $1.026(0.621-1.694)$ & $2.282^{c}(1.669-3.120)$ & $0.781(0.469-1.302)$ & $1.148(0.786-1.677)$ \\
\hline Middle & $1.241(0.899-1.714)$ & $1.979^{c}(1.583-2.475)$ & $0.775(0.508-1.183)$ & $0.997(0.723-1.375)$ \\
\hline Richer & $1.232^{\mathrm{a}}(0.977-1.553)$ & $1.704^{c}(1.448-2.006)$ & $0.928(0.652-1.32)$ & $0.968(0.734-1.277)$ \\
\hline \multicolumn{5}{|l|}{ Residence } \\
\hline Urban (ref) & 1 & 1 & 1 & 1 \\
\hline Rural & $1.092(0.864-1.381)$ & $0.927(0.785-1.093)$ & $0.748^{\mathrm{a}}(0.556-1.006)$ & $0.779^{\mathrm{b}}(0.624-0.974)$ \\
\hline \multicolumn{5}{|l|}{ Has health insurance } \\
\hline Yes (ref) & 1 & 1 & 1 & 1 \\
\hline No & $0.909(0.602-1.373)$ & $1.374^{\mathrm{b}}(1.011-1.867)$ & $0.320^{c}(0.246-0.417)$ & $0.829^{\mathrm{b}}(0.699-0.983)$ \\
\hline \multicolumn{5}{|l|}{ Religion } \\
\hline Other Christian (ref) & 1 & 1 & 1 & 1 \\
\hline Catholic & $1.078(0.811-1.433)$ & $1.048(0.849-1.295)$ & $0.824(0.57-1.19)$ & $0.891(0.676-1.173)$ \\
\hline Traditionalist/ none & $1.324(0.254-6.898)$ & $2.043(0.625-6.678)$ & $1.128(0.75-1.695)$ & $1.137(0.840-1.538)$ \\
\hline Islam & $1.188(0.915-1.542)$ & $1.474^{c}(1.240-1.753)$ & $0.527^{b}(0.297-0.932)$ & $1.109(0.781-1.575)$ \\
\hline \multicolumn{5}{|l|}{ Need permission for medical help } \\
\hline Not a big problem (ref) & 1 & 1 & 1 & 1 \\
\hline Big problem & $1.247(0.771-2.016)$ & $1.130(0.792-1.611)$ & $1.036(0.64-1.678)$ & $1.015(0.705-1.462)$ \\
\hline \multicolumn{5}{|l|}{ Need money for medical help } \\
\hline Not a big problem (ref) & 1 & 1 & 1 & 1 \\
\hline Big problem & $1.041(0.835-1.297)$ & $0.812^{C}(0.697-0.947)$ & $1.297^{\mathrm{a}}(0.999-1.682)$ & $1.051(0.868-1.273)$ \\
\hline \multicolumn{5}{|l|}{ Distance to health facility } \\
\hline Not a big problem (ref) & 1 & 1 & 1 & 1 \\
\hline
\end{tabular}


Table 2 Odds ratio of use of family planning services in Nigeria and Ghana (multinomial logistic regression) (Continued)

\begin{tabular}{|c|c|c|c|c|}
\hline \multirow[t]{4}{*}{ Background characteristics } & \multicolumn{2}{|l|}{ Nigeria } & \multicolumn{2}{|l|}{ Ghana } \\
\hline & Medium-access & Poor-access & Medium-access & Poor-access \\
\hline & \multicolumn{2}{|c|}{ Reference category: High access } & \multicolumn{2}{|c|}{ Reference category: High access } \\
\hline & $\operatorname{Exp} B(95 \%$ Cl) & $\operatorname{Exp} B(95 \% \mathrm{Cl})$ & $\operatorname{Exp} B(95 \%$ Cl) & $\operatorname{Exp} B(95 \% \mathrm{Cl})$ \\
\hline Big problem & $0.771^{\mathrm{a}}(0.571-1.042)$ & $1.109(0.904-1.360)$ & $1.237(0.908-1.685)$ & $1.164(0.923-1.466)$ \\
\hline \multicolumn{5}{|c|}{ Do not want to visit health facility alone } \\
\hline Not a big problem (ref) & 1 & 1 & 1 & 1 \\
\hline Big problem & $1.728^{\mathrm{b}}(1.112-2.685)$ & $1.407^{\mathrm{b}}(1.012-1.958)$ & $1.017(0.705-1.465)$ & $1.020(0.771-1.349)$ \\
\hline
\end{tabular}

\section{Heard family planning on radio last few months}

\section{Yes (ref) \\ No \\ $0.885(0.648-1.208)$}

Heard family planning on TV last few months

Yes (ref)

No

\section{Heard famil}

$$
\text { Yes (ref) }
$$

No

1

$0.790^{\mathrm{a}}(0.624-1.001)$

$0.790^{\mathrm{a}}(0.624$

\section{Region (Nigeria)}

South West (ref)

North Central

North East

North West

South East

South South

\section{Region}

Greater Accra (ref)

Western

Central

Volta

Eastern

Ashanti

Brong Ahafo

Northern

Upper East

Upper West

\section{Ethnicity (Nigeria)}

Yoruba (ref)
Other minorities
Fulani
Igbo
Hausa

\section{Ethnicity (Ghana)}

Akan (ref)

Ga/Dangme

Ewe $1.338^{\mathrm{a}}(0.961-1.864)$

$1.961(0.436-8.812)$

$2.134^{\mathrm{C}}(1.471-3.096)$

$4.820^{c}(2.139-10.861)$
11

$1.354^{\mathrm{b}}(1.066-1.720)$

1

$0.462^{c}(0.320-0.666)$

$0.162^{c}(0.070-0.376)$

$0.038^{\mathrm{c}}(0.016-0.086)$

$1.523^{b}(1.022-2.270)$

$1.100(0.780-1.550)$

1
1

$0.800^{\mathrm{b}}(0.643-0.997)$

1

$1.090(0.926-1.283)$

$1.105(0.934-1.308)$

$0.962(0.765-1.209)$

$1.574^{\mathrm{b}}(1.101-2.250)$

$0.425^{\mathrm{C}}(0.321-0.563)$

$1.390^{\mathrm{b}}(1.01-0$ 1.913)

$0.965(0.755-1.233)$
1

$1.742^{\mathrm{b}}(1.097-2.765)$

$0.754(0.463-1.229)$

$3.191^{\mathrm{C}}(1.890-5.389)$

$0.474^{\mathrm{C}}(0.273-0.825)$

$1.912^{c}(1.264-2.893)$

$1.826^{\mathrm{b}}(1.123-2.969)$

$1.623(0.835-3.155)$

$0.146^{\mathrm{c}}(0.045-0.473)$

$0.262^{\mathrm{b}}(0.090-0.769)$

1

$1.953^{\mathrm{C}}(1.566-2.436)$

$3.352^{c}(1.699-6.612)$

$1.922^{c}(1.448-2.551)$

$11.842^{\complement}(7.766-18.059)$
1

$1.023(0.846-1.236)$

1

$1.012(0.819-1.249)$

$0.710(0.458-1.103)$
1

$0.868(0.610-1.234)$

$0.691^{\mathrm{b}}(0.494-0.966)$

$0.499^{\mathrm{C}}(0.326-0.764)$

0.868 (0.617-1.223)

$1.103(0.808-1.505)$

$0.658^{\mathrm{b}}(0.451-0.960)$

$1.805^{\mathrm{b}}(1.083-3.006)$

0.709 (0.424-1.185)

$0.728(0.398-1.332)$
$1.294(0.775-2.159)$

$1.044(0.687-1.584)$
1

$1.142(0.790-1.650)$

$1.059(0.780-1.437)$ 
Table 2 Odds ratio of use of family planning services in Nigeria and Ghana (multinomial logistic regression) (Continued)

\begin{tabular}{|c|c|c|c|c|}
\hline \multirow[t]{4}{*}{ Background characteristics } & \multicolumn{2}{|l|}{ Nigeria } & \multicolumn{2}{|l|}{ Ghana } \\
\hline & Medium-access & Poor-access & Medium-access & Poor-access \\
\hline & \multicolumn{2}{|c|}{ Reference category: High access } & \multicolumn{2}{|c|}{ Reference category: High access } \\
\hline & Exp B $(95 \%$ Cl) & $\operatorname{Exp} B(95 \%$ Cl) & Exp B $(95 \%$ Cl) & Exp B $(95 \% \mathrm{Cl})$ \\
\hline Guan & - & - & $1.631(0.783-3.396)$ & $0.883(0.469-1.664)$ \\
\hline Mole-Dagbani & - & - & $1.184(0.754-1.860)$ & $1.061(0.754-1.493)$ \\
\hline Grusi & - & - & $0.942(0.490-1.811)$ & $0.682(0.422-1.103)$ \\
\hline Gurma & - & - & $0.948(0.523-1.720)$ & $0.883(0.563-1.385)$ \\
\hline Mande & - & - & $0.561(0.266-1.181)$ & $0.741(0.446-1.230)$ \\
\hline \multicolumn{5}{|c|}{ Attitude of the health workers } \\
\hline Not a big problem (ref) & 1 & 1 & - & - \\
\hline Big problem & $0.900(0.684-1.184)$ & $1.147(0.940-1.401)$ & - & - \\
\hline
\end{tabular}

${ }^{c} p \leq 0.01 ;{ }^{b} p \leq 0.05 ;{ }^{a} p \leq 0.10$ (two-tailed test of significance)

family planning cluster (in Nigeria $\mathrm{OR}=2.544,95 \% \mathrm{CI}$ : $1.907-3.395, p \leq 0.01$ and in Ghana OR $=1.527,95 \% \mathrm{CI}$ : $1.173-1.988, p \leq 0.01)$. Increased odds of having pooraccess to family planning services are found for women in Ghana who do not belong to white-collar workers but not among women who live in rural areas, and also not among women in any of the wealth quintiles. Higher odds of poor-access to family planning services are also found for women in Nigeria who belong to the serviceoccupational category $(\mathrm{OR}=1.283,95 \% \mathrm{CI}$ : $1.002-1.642$, $p \leq 0.05)$, compared with white-collar workers. The odds of poor-access are as much as three times higher among the poorest quintile $(95 \% \mathrm{CI}: 1.825-6.396, p \leq 0.01)$ than the richest quintile; and among those who have no insurance $(\mathrm{OR}=1.374,95 \% \mathrm{CI}: 1.011-1.867, p \leq 0.05)$ compared to those with insurance.

Table 3 shows the regression results on access to maternal health services in Nigeria and Ghana. In Nigeria's sample, women with primary or no education have higher odds to have poor-access $(\mathrm{OR}=1.387,95 \% \mathrm{CI}$ : $1.140-1.687, p \leq 0.01$ ) or low-access ( $\mathrm{OR}=1.786,95 \%$ CI: 1.247-2.557, $p \leq 0.01)$ to maternal health services. In Nigeria's sample, women who are not working have higher odds to belong to the cluster of poor-access maternal health services only (OR $=1.579,95 \%$ CI $1.081-$ $2.307, p \leq 0.01)$. Compared to women in the white-collar occupational group, women in other occupational categories in Nigeria also have higher odds to belong to the poor-access cluster. Women in other occupational categories in Nigeria also have higher odds to belong to the poor-access cluster. Women in all household wealth quintiles have higher odds to have high- or poor-access to maternal health services; women without insurance have higher odds to have high or poor-access to maternal health services. Results for Ghana show that women with primary $(\mathrm{OR}=1.38,95 \% \mathrm{CI}: 1.036-1.838, p \leq 0.05)$ or no education $(\mathrm{OR}=1.542,95 \% \mathrm{CI}$ : $1.115-2.132, p \leq$
0.01) have higher odds of poor-access to maternal health services. Only women in the agriculture occupational group have higher odds of high-access to maternal health services compared to women in the white-collar sector. Women without health insurance have higher odds of access to maternal health care services.

\section{Discussion}

As shown by our results, access to reproductive health services varies among women of reproductive age in Ghana and Nigeria. A large proportion of women in Ghana's and Nigeria's samples have poor access to family planning services. Most women do not have access to modern contraceptives. They use traditional birth control methods and do not have the means for needed services. These differences in access to maternal health services in both countries reflect a broader gap in health care use between women who access antenatal care at government hospitals for childbirth with a physician present and women who are not able to access such services. In particular, women in the low-access cluster are restricted to services at government health posts without skilled assistance during childbirth, or to services of antenatal care private vendors. This confirms that among women of reproductive age in Ghana and Nigeria, there is unequal access to reproductive health services.

This suggests a dysfunctional organization structure that creates constraints to use preventive and medical procedures provided by well-trained professionals $[4,18,22]$.

Our results show that educational attainment is associated with access to family planning and maternal health services. Low educational attainment reduces the ability to overcome access barriers, particularly to maternal health services. This finding supports similar results in other studies on the importance of education in improving access to reproductive health services [10-12, 23]. Our results indicate that some women with low 
Table 3 Odds ratio of use of maternal health service in Nigeria and Ghana: Ref: Higher access (multinomial logistic regression)

\begin{tabular}{|c|c|c|c|c|c|c|c|c|}
\hline \multirow{4}{*}{$\begin{array}{l}\text { Background } \\
\text { characteristics }\end{array}$} & \multicolumn{4}{|l|}{ Nigeria } & \multicolumn{4}{|l|}{ Ghana } \\
\hline & High-access & $\begin{array}{l}\text { Medium- } \\
\text { access }\end{array}$ & Low-access & Poor-access & High-access & $\begin{array}{l}\text { Medium- } \\
\text { access }\end{array}$ & Low-access & Poor-access \\
\hline & \multicolumn{4}{|c|}{ Reference category: Higher access } & \multicolumn{4}{|c|}{ Reference category: Higher access } \\
\hline & $\begin{array}{l}\operatorname{Exp} B \\
(95 \% \mathrm{Cl})\end{array}$ & $\begin{array}{l}\text { Exp B } \\
(95 \% \mathrm{Cl})\end{array}$ & $\begin{array}{l}\operatorname{Exp} B \\
(95 \% \mathrm{Cl})\end{array}$ & $\begin{array}{l}\text { Exp B } \\
(95 \% \mathrm{Cl})\end{array}$ & $\begin{array}{l}\operatorname{Exp~B} \\
(95 \% \mathrm{Cl})\end{array}$ & $\begin{array}{l}\operatorname{Exp} B \\
(95 \% \mathrm{Cl})\end{array}$ & $\begin{array}{l}\operatorname{Exp~B} \\
(95 \% \mathrm{Cl})\end{array}$ & $\begin{array}{l}\text { Exp B } \\
(95 \% \mathrm{Cl})\end{array}$ \\
\hline Maternal age & $\begin{array}{l}0.977^{c} \\
(0.963-0.992)\end{array}$ & $\begin{array}{l}0.980^{c} \\
(0.967-0.994)\end{array}$ & $\begin{array}{l}0.956^{c} \\
(0.930-0.982)\end{array}$ & $\begin{array}{l}0.965^{c}(0.950- \\
0.980)\end{array}$ & $\begin{array}{l}0.977^{\mathrm{b}} \\
(0.957-0.997)\end{array}$ & $\begin{array}{l}0.984^{\mathrm{a}} \\
(0.963-1.005)\end{array}$ & $\begin{array}{l}1.020 \\
(0.995-1.047)\end{array}$ & $\begin{array}{l}0.961^{c} \\
(0.940-0.983)\end{array}$ \\
\hline $\begin{array}{l}\text { Number of children } \\
\text { alive }\end{array}$ & $\begin{array}{l}1.101^{c} \\
(1.045-1.16)\end{array}$ & $\begin{array}{l}1.087^{c} \\
(1.033-1.145)\end{array}$ & $\begin{array}{l}1.113^{b} \\
(1.016-1.220)\end{array}$ & $\begin{array}{l}1.126^{c} \\
(1.067-1.188)\end{array}$ & $\begin{array}{l}1.105^{\mathrm{b}} \\
(1.016-1.201)\end{array}$ & $\begin{array}{l}1.088^{\mathrm{a}} \\
(0.998-1.185)\end{array}$ & $\begin{array}{l}1.000 \\
(0.899-1.113)\end{array}$ & $\begin{array}{l}1.239^{c} \\
(1.134-1.353)\end{array}$ \\
\hline \multicolumn{9}{|l|}{ Marital status } \\
\hline Married (ref) & 1 & 1 & 1 & 1 & 1 & 1 & 1 & 1 \\
\hline Never married & $\begin{array}{l}1.081 \\
(0.722-1.619)\end{array}$ & $\begin{array}{l}0.894 \\
(0.600-1.332)\end{array}$ & $\begin{array}{l}1.550 \\
(0.676-3.551)\end{array}$ & $\begin{array}{l}1.382 \\
(0.922-2.072)\end{array}$ & $\begin{array}{l}0.981 \\
(0.699-1.377)\end{array}$ & $\begin{array}{l}0.713^{\mathrm{a}} \\
(0.495-1.026)\end{array}$ & $\begin{array}{l}0.970 \\
(0.628-1.498)\end{array}$ & $\begin{array}{l}0.880 \\
(0.604-1.281)\end{array}$ \\
\hline $\begin{array}{l}\text { Widowed/ } \\
\text { separated/divorced }\end{array}$ & $\begin{array}{l}1.252 \\
(0.871-1.800)\end{array}$ & $\begin{array}{l}1.055 \\
(0.729-1.527)\end{array}$ & $\begin{array}{l}1.248 \\
(0.634-2.459)\end{array}$ & $\begin{array}{l}1.084 \\
(0.740-1.588)\end{array}$ & $\begin{array}{l}0.978 \\
(0.676-1.413)\end{array}$ & $\begin{array}{l}0.869 \\
(0.59-1.278)\end{array}$ & $\begin{array}{l}0.803 \\
(0.477-1.350)\end{array}$ & $\begin{array}{l}0.880 \\
(0.591-1.310)\end{array}$ \\
\hline \multicolumn{9}{|l|}{ Maternal Education } \\
\hline $\begin{array}{l}\text { Secondary/ Higher } \\
\text { (ref) }\end{array}$ & 1 & 1 & 1 & 1 & 1 & 1 & 1 & 1 \\
\hline No education & $\begin{array}{l}1.145 \\
(0.902-1.454)\end{array}$ & $\begin{array}{l}0.690^{c} \\
(0.524-0.909)\end{array}$ & $\begin{array}{l}1.849^{c} \\
(1.247-2.742)\end{array}$ & $\begin{array}{l}1.431^{\mathrm{C}} \\
(1.132-1.809)\end{array}$ & $\begin{array}{l}0.961 \\
(0.702-1.316)\end{array}$ & $\begin{array}{l}0.962 \\
(0.691-1.341)\end{array}$ & $\begin{array}{l}0.680^{\mathrm{a}} \\
(0.43-1.076)\end{array}$ & $\begin{array}{l}1.542^{\mathrm{C}} \\
(1.115-2.132)\end{array}$ \\
\hline Primary & $\begin{array}{l}1.034 \\
(0.855-1.251)\end{array}$ & $\begin{array}{l}0.921 \\
(0.763-1.111)\end{array}$ & $\begin{array}{l}1.786^{c} \\
(1.247-2.557)\end{array}$ & $\begin{array}{l}1.387^{c} \\
(1.140-1.687)\end{array}$ & $\begin{array}{l}0.975 \\
(0.742-1.280)\end{array}$ & $\begin{array}{l}1.202 \\
(0.914-1.580)\end{array}$ & $\begin{array}{l}0.594^{\mathrm{b}} \\
(0.398-0.886)\end{array}$ & $\begin{array}{l}1.380^{\mathrm{b}} \\
(1.036-1.838)\end{array}$ \\
\hline \multicolumn{9}{|l|}{ Maternal Occupation } \\
\hline White collar (ref) & 1 & 1 & 1 & 1 & 1 & 1 & 1 & 1 \\
\hline Not working & $\begin{array}{l}1.052 \\
(0.779-1.421)\end{array}$ & $\begin{array}{l}1.097 \\
(0.847-1.422)\end{array}$ & $\begin{array}{l}1.754 \\
(0.752-4.093)\end{array}$ & $\begin{array}{l}1.579^{\mathrm{b}} \\
(1.081-2.307)\end{array}$ & $\begin{array}{l}1.074 \\
(0.644-1.79)\end{array}$ & $\begin{array}{l}0.771 \\
(0.474-1.254)\end{array}$ & $\begin{array}{l}0.889 \\
(0.535-1.476)\end{array}$ & $\begin{array}{l}1.076 \\
(0.500-2.313)\end{array}$ \\
\hline $\begin{array}{l}\text { Services and } \\
\text { manual }\end{array}$ & $\begin{array}{l}1.492^{c} \\
(1.109-2.006)\end{array}$ & $\begin{array}{l}1.215 \\
(0.940-1.570)\end{array}$ & $\begin{array}{l}2.422^{\mathrm{b}} \\
(1.032-5.686)\end{array}$ & $\begin{array}{l}1.719^{c} \\
(1.172-2.522)\end{array}$ & $\begin{array}{l}1.168 \\
(0.699-1.952)\end{array}$ & $\begin{array}{l}0.782 \\
(0.479-1.276)\end{array}$ & $\begin{array}{l}0.437^{c} \\
(0.252-0.757)\end{array}$ & $\begin{array}{l}1.141 \\
(0.527-2.468)\end{array}$ \\
\hline Sales & $\begin{array}{l}1.289^{\mathrm{a}} \\
(0.979-1.698)\end{array}$ & $\begin{array}{l}1.300^{\mathrm{b}} \\
(1.032-1.638)\end{array}$ & $\begin{array}{l}2.729^{b} \\
(1.198-6.219)\end{array}$ & $\begin{array}{l}2.001^{\mathrm{C}} \\
(1.395-2.871)\end{array}$ & $\begin{array}{l}0.824 \\
(0.511-1.329)\end{array}$ & $\begin{array}{l}0.576^{\mathrm{b}} \\
(0.368-0.903)\end{array}$ & $\begin{array}{l}0.628^{b} \\
(0.4-0.987)\end{array}$ & $\begin{array}{l}0.779 \\
(0.371-1.636)\end{array}$ \\
\hline Agriculture & $\begin{array}{l}1.697^{c} \\
(1.187-2.428)\end{array}$ & $\begin{array}{l}1.298 \\
(0.922-1.826)\end{array}$ & $\begin{array}{l}4.253^{c} \\
(1.741-10.392)\end{array}$ & $\begin{array}{l}1.667^{\mathrm{b}} \\
(1.076-2.584)\end{array}$ & $\begin{array}{l}1.781^{b} \\
(1.022-3.104)\end{array}$ & $\begin{array}{l}0.735 \\
(0.426-1.266)\end{array}$ & $\begin{array}{l}0.539^{\mathrm{a}} \\
(0.277-1.05)\end{array}$ & $\begin{array}{l}1.226 \\
(0.561-2.682)\end{array}$ \\
\hline
\end{tabular}

\section{Household Wealth}

$\begin{array}{lllllllll}\text { Richest (ref) } & 1 & 1 & 1 & 1 & 1 & 1 & 1 & 1 \\ \text { Poorest } & 4.726^{c} & 1.531 & 3.230^{c} & 6.592^{c} & 3.732^{c} & 3.826^{c} & 1.889 & 20.631^{c} \\ & (2.982-7.489) & (0.855-2.740) & (1.668-6.255) & (4.247-10.233) & (2.171-6.415) & (2.16-6.775) & (0.863-4.135) & (10.086-42.199) \\ \text { Poorer } & 2.750^{c} & 1.104 & 1.580^{\mathrm{a}} & 2.408^{c} & 2.514^{c} & 2.351^{c} & 1.679^{\mathrm{a}} & 10.228^{c} \\ & (2.018-3.748) & (0.796-1.533) & (0.921-2.711) & (1.75-3.312) & (1.605-3.938) & (1.479-3.737) & (0.949-2.97) & (5.352-19.544) \\ \text { Middle } & 1.829^{c} & 0.832(0.657- & 1.268 & 1.810^{c} & 1.967^{c} & 1.684^{c} & 1.077 & 6.376^{c} \\ & (1.433-2.334) & 1.054) & (0.795-2.023) & (1.401-2.338) & (1.363-2.838) & (1.153-2.459) & (0.705-1.645) & (3.508-11.587) \\ \text { Richer } & 1.476^{c} & 0.733 c & 0.665^{\mathrm{a}} & 1.237^{\mathrm{b}} & 1.263 & 1.354^{\mathrm{a}} & 0.627^{c} & 1.328 \\ & (1.221-1.785) & (0.616-0.873) & (0.433-1.022) & (1.006-1.522) & (0.926-1.723) & (0.991-1.851) & (0.447-0.881) & (0.713-2.474)\end{array}$

\section{Residence}

$\begin{array}{lllllllll}\text { Urban (ref) } & 1 & 1 & 1 & 1 & 1 & 1 & 1 & 1 \\ \text { Rural } & 0.654^{\mathrm{c}} & 1.086 & 0.539^{\mathrm{c}} & 0.817^{\mathrm{b}} & 1.149 & 1_{1.303^{\mathrm{a}}} & 0.646^{\mathrm{b}} & 2.139^{\mathrm{c}} \\ & (0.550-0.779) & (0.914-1.290) & (0.384-0.757) & (0.678-0.985) & (0.889-1.483) & (0.999-1.699) & (0.453-0.921) & (1.600-2.86)\end{array}$

\section{Has health insurance}

$\begin{array}{lllllllll}\text { Yes (ref) } & 1 & 1 & 1 & 1 & 1 & 1 & 1 & 1 \\ \text { No } & 1.167 & 0.789 & 0.563 & 1.570^{\mathrm{a}} & 1.146 & 1.260^{\mathrm{b}} & 1.209 & 1.888^{\mathrm{c}} \\ & (0.770-1.770) & (0.574-1.085) & (0.274-1.156) & (0.926-2.661) & (0.923-1.422) & (1.009-1.573) & (0.919-1.590) & (1.500-2.376)\end{array}$


Table 3 Odds ratio of use of maternal health service in Nigeria and Ghana: Ref: Higher access (multinomial logistic regression) (Continued)

\begin{tabular}{|c|c|c|c|c|c|c|c|c|}
\hline \multirow{4}{*}{$\begin{array}{l}\text { Background } \\
\text { characteristics }\end{array}$} & \multicolumn{4}{|l|}{ Nigeria } & \multicolumn{4}{|l|}{ Ghana } \\
\hline & High-access & $\begin{array}{l}\text { Medium- } \\
\text { access }\end{array}$ & Low-access & Poor-access & High-access & $\begin{array}{l}\text { Medium- } \\
\text { access }\end{array}$ & Low-access & Poor-access \\
\hline & \multicolumn{4}{|c|}{ Reference category: Higher access } & \multicolumn{4}{|c|}{ Reference category: Higher access } \\
\hline & $\begin{array}{l}\text { Exp B } \\
(95 \% \mathrm{Cl})\end{array}$ & $\begin{array}{l}\text { Exp B } \\
(95 \% \mathrm{Cl})\end{array}$ & $\begin{array}{l}\text { Exp B } \\
(95 \% \mathrm{Cl})\end{array}$ & $\begin{array}{l}\text { Exp B } \\
(95 \% \mathrm{Cl})\end{array}$ & $\begin{array}{l}\text { Exp B } \\
(95 \% \mathrm{Cl})\end{array}$ & $\begin{array}{l}\text { Exp B } \\
(95 \% \mathrm{Cl})\end{array}$ & $\begin{array}{l}\text { Exp B } \\
(95 \% \mathrm{Cl})\end{array}$ & $\begin{array}{l}\text { Exp B } \\
(95 \% \mathrm{Cl})\end{array}$ \\
\hline $\begin{array}{l}\text { Other Christian } \\
\text { (ref) }\end{array}$ & 1 & 1 & 1 & 1 & 1 & 1 & 1 & 1 \\
\hline Catholic & $\begin{array}{l}1.244^{\mathrm{a}} \\
(0.970-1.595)\end{array}$ & $\begin{array}{l}1.582^{\mathrm{a}} \\
(1.269-1.973)\end{array}$ & $\begin{array}{l}1.355 \\
(0.847-2.166)\end{array}$ & $\begin{array}{l}0.952 \\
(0.709-1.278)\end{array}$ & $\begin{array}{l}0.710^{b} \\
(0.505-0.998)\end{array}$ & $\begin{array}{l}0.698^{\mathrm{b}} \\
(0.492-0.99)\end{array}$ & $\begin{array}{l}0.496^{c} \\
(0.300-0.820)\end{array}$ & $\begin{array}{l}0.679^{b} \\
(0.470-0.980)\end{array}$ \\
\hline vTraditionalist/ none & $\begin{array}{l}0.643 \\
(0.249-1.661)\end{array}$ & $\begin{array}{l}0.470 \\
(0.165-1.340)\end{array}$ & $\begin{array}{l}0.379 \\
(0.034-4.263)\end{array}$ & $\begin{array}{l}0.470 \\
(0.154-1.432)\end{array}$ & $\begin{array}{l}1.031 \\
(0.719-1.478)\end{array}$ & $\begin{array}{l}0.763 \\
(0.524-1.113)\end{array}$ & $\begin{array}{l}0.825 \\
(0.500-1.363)\end{array}$ & $\begin{array}{l}0.781 \\
(0.528-1.154)\end{array}$ \\
\hline Islam & $\begin{array}{l}0.992 \\
(0.810-1.214)\end{array}$ & $\begin{array}{l}0.916 \\
(0.760-1.104)\end{array}$ & $\begin{array}{l}1.429^{\mathrm{a}} \\
(0.971-2.105)\end{array}$ & $\begin{array}{l}0.976 \\
(0.783-1.215)\end{array}$ & $\begin{array}{l}1.112 \\
(0.644-1.921)\end{array}$ & $\begin{array}{l}1.294 \\
(0.724-2.314)\end{array}$ & $\begin{array}{l}2.472^{c} \\
(1.263-4.837)\end{array}$ & $\begin{array}{l}2.244^{c} \\
(1.351-3.726)\end{array}$ \\
\hline
\end{tabular}

\section{Need permission for medical help}

$\begin{array}{lllllllll}\begin{array}{l}\text { Not a big problem } \\ \text { (ref) }\end{array} & 1 & 1 & 1 & 1 & 1 & 1 & 1 & 1 \\ \text { Big problem } & 0.990 & 0.781 & 0.620 & 1.016 & 1.097 & 0.816 & 0.478^{\mathrm{b}} & 0.840 \\ & (0.709-1.384) & (0.540-1.128) & (0.312-1.232) & (0.730-1.415) & (0.711-1.691) & (0.525-1.267) & (0.23-0.993) & (0.532-1.328)\end{array}$

\section{Need money for medical help}

$\begin{array}{lllllllll}\begin{array}{l}\text { Not a big problem } \\ \text { (ref) }\end{array} & 1 & 1 & 1 & 1 & 1 & 1 & 1 & 1 \\ \text { Big problem } & 0.996 & 0.879 & 1.033 & 1.304^{c} & 0.999 & 1.239^{\text {a }} & 0.668^{\text {b }} & 0.889 \\ & (0.843-1.178) & (0.744-1.037) & (0.768-1.389) & (1.100-1.547) & (0.794-1.256) & (0.977-1.572) & (0.487-0.916) & (0.693-1.14)\end{array}$

\section{Distance to health facility}

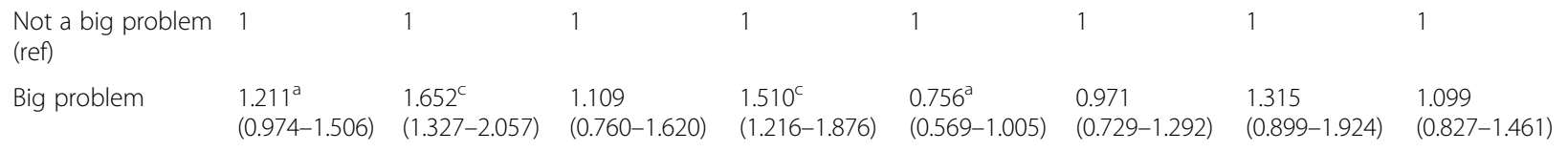

\section{Do not want to visit health facility alone}

$\begin{array}{lllllllll}\begin{array}{l}\text { Not a big problem } \\ \text { (ref) }\end{array} & 1 & 1 & 1 & 1 & 1 & 1 & 1 & 1 \\ \text { Big problem } & 0.869 & 0.982 & 0.859 & 0.791 & 1.020 & 0.932 & 0.930 & 1.431^{b} \\ & (0.632-1.196) & (0.714-1.351) & (0.471-1.565) & (0.566-1.106) & (0.728-1.427) & (0.658-1.321) & (0.592-1.462) & (1.02-2.008)\end{array}$

\section{Heard family planning on radio last few months}

$\begin{array}{lll}\text { Yes (ref) } & 1 & 1 \\ \text { No } & 1.008 & 0.776^{\mathrm{b}} \\ & (0.797-1.275) & (0.612-0.983) \\ \text { Heard family planning on TV last few months }\end{array}$

\section{Heard family planning on TV last few months}

$\begin{array}{lllllllll}\text { Yes (ref) } & 1 & 1 & 1 & 1 & 1 & 1 & 1 & 1 \\ \text { No } & 0.917 & 0.934 & 0.928 & 1.075 & 1.090 & 0.852 & 0.773 & 0.923 \\ & (0.768-1.095) & (0.785-1.112) & (0.670-1.286) & (0.896-1.290) & (0.848-1.399) & (0.656-1.105) & (0.554-1.077) & (0.704-1.21)\end{array}$

\section{Heard family planning in print last few months}

$\begin{array}{lllllllll}\text { Yes (ref) } & 1 & 1 & 1 & 1 & 1 & 1 & 1 & 1 \\ \text { No } & 0.861 & 0.877 & 1.384 & 1.068 & 1.733^{b} & 0.930 & 1.526 & 1.195 \\ & (0.708-1.047) & (0.729-1.053) & (0.885-2.165) & (0.861-1.324) & (1.042-2.883) & (0.588-1.473) & (0.909-2.562) & (0.614-2.324)\end{array}$

\section{Region (Nigeria)}

$\begin{array}{lllll}\text { South West (ref) } & 1 & 1 & 1 & 1 \\ \text { North Central } & 0.377^{c} & 0.335^{c} & 0.666 & 0.343^{c} \\ & (0.286-0.499) & (0.264-0.425) & (0.381-1.166) & (0.250-0.470) \\ \text { North East } & 0.427^{c} & 0.061^{c} & 1.162(0.632- & 0.596^{c}(0.418-\end{array}$


Table 3 Odds ratio of use of maternal health service in Nigeria and Ghana: Ref: Higher access (multinomial logistic regression) (Continued)

\begin{tabular}{|c|c|c|c|c|c|c|c|c|}
\hline \multirow{4}{*}{$\begin{array}{l}\text { Background } \\
\text { characteristics }\end{array}$} & \multicolumn{4}{|l|}{ Nigeria } & \multicolumn{4}{|l|}{ Ghana } \\
\hline & High-access & $\begin{array}{l}\text { Medium- } \\
\text { access }\end{array}$ & Low-access & Poor-access & High-access & $\begin{array}{l}\text { Medium- } \\
\text { access }\end{array}$ & Low-access & Poor-access \\
\hline & \multicolumn{4}{|c|}{ Reference category: Higher access } & \multicolumn{4}{|c|}{ Reference category: Higher access } \\
\hline & $\begin{array}{l}\operatorname{Exp~B} \\
(95 \% \mathrm{Cl}) \\
\end{array}$ & $\begin{array}{l}\operatorname{Exp~B} \\
(95 \% \mathrm{Cl}) \\
\end{array}$ & $\begin{array}{l}\operatorname{Exp~B} \\
(95 \% \mathrm{Cl})\end{array}$ & $\begin{array}{l}\operatorname{Exp~B} \\
(95 \% \mathrm{Cl})\end{array}$ & $\begin{array}{l}\text { Exp B } \\
(95 \% \text { Cl) }\end{array}$ & $\begin{array}{l}\text { Exp B } \\
(95 \% \mathrm{Cl}) \\
\end{array}$ & $\begin{array}{l}\operatorname{Exp~B} \\
(95 \% \mathrm{Cl})\end{array}$ & $\begin{array}{l}\operatorname{Exp~B} \\
(95 \% \mathrm{Cl})\end{array}$ \\
\hline & $(0.302-0.603)$ & $(0.039-0.096)$ & $2.136)$ & $0.850)$ & & & & \\
\hline North West & $\begin{array}{l}0.206^{c} \\
(0.145-0.292)\end{array}$ & $\begin{array}{l}0.030^{c} \\
(0.020-0.046)\end{array}$ & $\begin{array}{l}0.344^{c}(0.178- \\
0.665)\end{array}$ & $\begin{array}{l}0.346^{c} \\
(0.242-0.492)\end{array}$ & - & - & - & - \\
\hline South East & $\begin{array}{l}1.112 \\
(0.736-1.681)\end{array}$ & $\begin{array}{l}0.771 \\
(0.554-1.073)\end{array}$ & $\begin{array}{l}1.712 \\
(0.635-4.618)\end{array}$ & $\begin{array}{l}0.961 \\
(0.567-1.629)\end{array}$ & - & - & - & - \\
\hline South South & $\begin{array}{l}1.031 \\
(0.767-1.387)\end{array}$ & $\begin{array}{l}0.319^{c} \\
(0.244-0.417)\end{array}$ & $\begin{array}{l}0.248^{\mathrm{a}} \\
(0.107-0.577)\end{array}$ & $\begin{array}{l}1.401^{\mathrm{b}} \\
(1.019-1.928)\end{array}$ & - & - & - & - \\
\hline
\end{tabular}

\section{Region}

Greater Accra (ref) -

Western

Central

Volta

Region (continued)

Eastern

Ashanti

Brong Ahafo

Northern

Upper East

Upper West

\section{Ethnicity (Nigeria)}

$\begin{array}{lllll}\text { Yoruba (ref) } & 1 & 1 & 1 & \\ \text { Other minorities } & 0.541^{c} & 0.987 & 1.223 & 0.816 \\ & (0.412-0.71) & (0.780-1.249) & (0.677-2.208) & (0.603-1.105) \\ \text { Fulani } & 0.681 & 0.868(0.400- & 0.897 & 0.881 \\ & (0.405-1.143) & 1.883) & (0.376-2.139) & (0.529-1.466) \\ \text { Igbo } & 0.759 & 1.808^{c} & 1.014 & 0.495^{c} \\ & (0.519-1.109) & (1.336-2.447) & (0.378-2.722) & (0.305-0.803) \\ \text { Hausa } & 0.862 & 1.177(0.760- & 2.231^{\mathrm{b}} & 1.416^{\mathrm{a}} \\ & (0.59-1.259) & 1.822) & (1.122-4.438) & (0.965-2.078)\end{array}$

\section{Ethnicity (Ghana)}

Akan (ref)

Ga/Dangme

Ewe

Guan

$\begin{array}{llll}1 & 1 & 1 & 1 \\ 0.369^{c} & 1.444^{\mathrm{a}} & 1.013 & 1.340 \\ (0.237-0.576) & (0.948-2.198) & (0.625-1.64) & (0.767-2.342) \\ 0.994 & 1.596^{\mathrm{b}} & 1.171 & 2.101^{\mathrm{c}} \\ (0.666-1.483) & (1.036-2.459) & (0.721-1.904) & (1.211-3.648) \\ 1.133 & 0.480^{c} & 0.411^{c} & 0.834 \\ (0.695-1.849) & (0.274-0.841) & (0.208-0.811) & (0.452-1.539)\end{array}$

$\begin{array}{llll}0.444^{c} & 1.228 & 0.570^{\mathrm{b}} & 1.253 \\ (0.288-0.684) & (0.804-1.874) & (0.333-0.977) & (0.733-2.142) \\ 0.900 & 2.429^{c} & 1.660^{c} & 1.093 \\ (0.633-1.279) & (1.681-3.511) & (1.131-2.435) & (0.633-1.888) \\ 0.648^{\mathrm{a}} & 1.705^{\mathrm{b}} & 1.690^{\mathrm{b}} & 0.871 \\ (0.415-1.01) & (1.075-2.705) & (1.014-2.818) & (0.478-1.587) \\ 2.036^{\mathrm{b}} & 0.753 & 0.225^{\mathrm{b}} & 2.962^{c} \\ (1.163-3.564) & (0.382-1.484) & (0.071-0.711) & (1.489-5.891) \\ 3.661^{c} & 1.161 & 0.762 & 0.665 \\ (1.92-6.98) & (0.542-2.486) & (0.249-2.329) & (0.287-1.541) \\ 1.789 & 7.466 c & 0.348 & 2.861^{\mathrm{b}} \\ (0.713-4.489) & (3.195-17.443) & (0.033-3.654) & (1.079-7.586)\end{array}$

$\begin{array}{llll}(0.713-4.489) & (3.195-17.443) & (0.033-3.654) & (1.079-7.586)\end{array}$

$\begin{array}{llll}1 & 1 & 1 & 1 \\ 1.592^{\mathrm{b}} & 1.286 & 1.456 & 1.319 \\ (1.068-2.374) & (0.813-2.035) & (0.892-2.376) & (0.776-2.243) \\ 0.647^{\mathrm{b}} & 1.469^{\mathrm{b}} & 1.633^{\mathrm{b}} & 1.154 \\ (0.438-0.957) & (1.019-2.119) & (1.076-2.477) & (0.764-1.743) \\ 1.544 & 3.760^{c} & 7.107^{c} & 1.639 \\ (0.676-3.525) & (1.678-8.424) & (3.037-16.632) & (0.674-3.988)\end{array}$


Table 3 Odds ratio of use of maternal health service in Nigeria and Ghana: Ref: Higher access (multinomial logistic regression) (Continued)

\begin{tabular}{|c|c|c|c|c|c|c|c|c|}
\hline \multirow{4}{*}{$\begin{array}{l}\text { Background } \\
\text { characteristics }\end{array}$} & \multicolumn{4}{|l|}{ Nigeria } & \multicolumn{4}{|l|}{ Ghana } \\
\hline & High-access & $\begin{array}{l}\text { Medium- } \\
\text { access }\end{array}$ & Low-access & Poor-access & High-access & $\begin{array}{l}\text { Medium- } \\
\text { access }\end{array}$ & Low-access & Poor-access \\
\hline & \multicolumn{4}{|c|}{ Reference category: Higher access } & \multicolumn{4}{|c|}{ Reference category: Higher access } \\
\hline & $\begin{array}{l}\operatorname{Exp} B \\
(95 \% \mathrm{Cl})\end{array}$ & $\begin{array}{l}\operatorname{Exp~B} \\
(95 \% \mathrm{Cl})\end{array}$ & $\begin{array}{l}\operatorname{Exp~B} \\
(95 \% \mathrm{Cl})\end{array}$ & $\begin{array}{l}\operatorname{Exp~B} \\
(95 \% \mathrm{Cl})\end{array}$ & $\begin{array}{l}\operatorname{Exp~B} \\
(95 \% \mathrm{Cl})\end{array}$ & $\begin{array}{l}\operatorname{Exp~B} \\
(95 \% \mathrm{Cl})\end{array}$ & $\begin{array}{l}\operatorname{Exp~B} \\
(95 \% \mathrm{Cl})\end{array}$ & $\begin{array}{l}\operatorname{Exp~B} \\
(95 \% \mathrm{Cl})\end{array}$ \\
\hline Mole-Dagbani & - & - & - & - & $\begin{array}{l}0.754 \\
(0.494-1.150)\end{array}$ & $\begin{array}{l}1.391^{\mathrm{a}} \\
(0.927-2.088)\end{array}$ & $\begin{array}{l}1.605^{\mathrm{a}} \\
(0.955-2.698)\end{array}$ & $\begin{array}{l}1.056 \\
(0.676-1.649)\end{array}$ \\
\hline Grusi & - & - & - & - & $\begin{array}{l}0.850 \\
(0.463-1.561)\end{array}$ & $\begin{array}{l}1.102 \\
(0.587-2.07)\end{array}$ & $\begin{array}{l}1.056 \\
(0.441-2.527)\end{array}$ & $\begin{array}{l}1.113 \\
(0.564-2.199)\end{array}$ \\
\hline Gurma & - & - & - & - & $\begin{array}{l}0.795 \\
(0.438-1.443)\end{array}$ & $\begin{array}{l}1.249 \\
(0.658-2.373)\end{array}$ & $\begin{array}{l}0.929 \\
(0.33-2.615)\end{array}$ & $\begin{array}{l}1.438 \\
(0.792-2.613)\end{array}$ \\
\hline Mande & - & - & - & - & $\begin{array}{l}0.604 \\
(0.318-1.149)\end{array}$ & $\begin{array}{l}1.257 \\
(0.651-2.425)\end{array}$ & $\begin{array}{l}0.840 \\
(0.34-2.071)\end{array}$ & $\begin{array}{l}1.252 \\
(0.627-2.497)\end{array}$ \\
\hline \multicolumn{9}{|c|}{ Attitude of the health workers } \\
\hline $\begin{array}{l}\text { Not a big problem } \\
\text { (ref) }\end{array}$ & 1 & 1 & 1 & 1 & - & - & - & - \\
\hline Big problem & $\begin{array}{l}1.707^{c} \\
(1.314-2.217)\end{array}$ & $\begin{array}{l}1.361^{c} \\
(1.098-1.686)\end{array}$ & $\begin{array}{l}0.809 \\
(0.450-1.455)\end{array}$ & $\begin{array}{l}2.495^{\complement} \\
(1.748-3.56)\end{array}$ & - & - & - & - \\
\hline
\end{tabular}

${ }^{c} p \leq 0.01 ;{ }^{\mathrm{b}} p \leq 0.05 ;{ }^{\mathrm{a}} p \leq 0.10$ (two-tailed test of significance

education intend to use contraceptives later while others use traditional contraceptive methods of family planning. Notably, the cluster with poor access to family planning services for a large part consists of women who have no intention of future contraceptive use. Lower-educated women seem to be less able to act on their intentions due to difficulties in overcoming access barriers or limited knowledge about the benefits of family planning [24]. The connection between education and socioeconomic status could also explain this observation because low education attainment usually implies less access to resources $[10,25,26]$. This result further confirms what is known about the educational level as an indirect predictor of access to health care services [9].

Our results suggest that wealth/finance related inequality in access to reproductive health services is prominent in both Nigeria and Ghana. Considering finance-related inequality between the two countries, we find that women without insurance coverage in Ghana are less likely to access family planning services. This is dissimilar when compared to women without insurance in Nigeria; women in Nigeria who have poor-access to family planning opt for services such as traditional methods of contraception. These findings are consistent with other studies on the use of family planning services in the two countries and other parts of Africa [10]. This can be partially attributed to the inaccessibility of family planning services due to a cost-reducing scheme, which inadvertently increases the preference for traditional contraceptives among some women [27]. Another study also found a situation similar to Ghana among women in Burkina Faso and concluded that affordability of insurance premium varies by household income [13]. The poor access to reproductive health services in any of the wealth quintiles in Nigeria is expected considering the lack of insurance. The low coverage of insurance schemes such as the NHIS, particularly among informal workers or uneducated women, magnifies the effect of household wealth $[17,18]$.

There is an association between maternal occupation and access to maternal health services in both countries. Other studies have also reported associations between care use and occupation [14, 22, 23]. However, where associations between maternal occupation and access to reproductive health services are observable, disparity by type of livelihood is not unusual [22]. We observe such differences between Ghana and Nigeria as well. In particular, the group of white-collar workers seems to have better access to family planning services in Ghana but no such differences are found for maternal care. The results for Nigeria are just the opposite; occupation does not explain poor access to family planning services in Nigeria but white-collar workers seem to have access to maternal care. In Nigeria, the cost of maternal health services has to be endured by women themselves while in Ghana, these services are available to women through the free maternal care policy $[28,29]$. Out-of-pocket payments for health have been consistently high in Nigeria compared to those in Ghana while insurance coverage is better in Ghana, particularly in the informal sector $[27,30]$. 
This study has some limitations that need to be acknowledged. There was not much variation in some response variables and they had to be excluded from the analysis. The inclusion of country-specific variables helps to better reflect the women's situation but this also creates some dissimilarities in the countries' analytical models.

\section{Conclusion}

This study provided evidence on inequalities in access to reproductive health services in Ghana and Nigeria. A key observation is the varied composition of services available for use at different access levels. Several imperative factors contribute to inequality in access to these services. After controlling for the effects of maternal-related variables, findings showed significant inequalities by educational attainment, household wealth, insurance status and woman's occupational type. Much of the inequality in access to family planning services that are seen in Nigeria and Ghana is related to education. The contribution of household wealth and insurance status in creating unequal access was also evidenced in the study. Health programs, which seek to stimulate the use of reproductive health services in Ghana and Nigeria, could take into account the variation in access reported in this study to assure the user-centeredness of these programs. It is important to identify and prioritize services for the needs of vulnerable groups.

\section{Supplementary information}

Supplementary information accompanies this paper at https://doi.org/10. 1186/s12889-020-08724-3.

Additional file 1.

\section{Acknowledgments}

Not applicable.

\section{Authors' contributions}

$\mathrm{JO}$ and MP designed the study. $\mathrm{JO}$ analyzed the data and drafted the manuscript. JO, MP, and WG subsequently revised the manuscript and approved the final draft for submission. The author(s) read and approved the final manuscript.

\section{Funding}

Not applicable.

\section{Availability of data and materials}

The data used for this study can be accessed through the following link: https://dhsprogram.com/data/dataset_admin/login_main.cfm?CFID=1555476 9\&CFTOKEN=6d6c1572de0c435a-5BA70A19-BD9F-EDBD-DBCOBB52339CAF30

\section{Ethics approval and consent to participate}

Administrative permissions were required and obtained from the Demographic and Health Survey program to access the data used in this study. We made use of secondary DHS data from Ghana and Nigeria. Therefore, our study did not require a formal ethics approval and the study complied with Maastricht University code of ethics for research in the social and behavioral sciences involving human participants, and also with the national guidelines (nietWMOplichtig onderzoek or the Medical Research Involving Human Subjects Act (WMO) Link: https://www.maastrichtuniversity. $\mathrm{nl} /$ research/integrity-ethics/ethics-review and https://nwmostudies.nl/nwmo/
Consent for publication

Not applicable.

\section{Competing interests}

The authors declare that they have no competing interests.

\section{Author details}

1Department of Health Services Research; CAPHRI, Maastricht University Medical Center; Faculty of Health, Medicine and Life Sciences; Maastricht University, PO Box 616, 6200MD, Maastricht, The Netherlands. ${ }^{2}$ United Nations University-Maastricht Economic and Social Research Institute on Innovation and Technology, Maastricht, The Netherlands.

Received: 23 October 2019 Accepted: 16 April 2020

Published online: 23 April 2020

\section{References}

1. Cook RJ. International human rights and women's reproductive health. Stud Fam Plan. 1993:24(2):73-86.

2. Braveman $P$, Tarimo E. Social inequalities in health within countries: not only an issue for affluent nations. Soc Sci Med. 2002;54(11):1621-35.

3. Canning D, Schultz TP. The economic consequences of reproductive health and family planning. Lancet. 2012;380(9837):165-71.

4. Koblinsky MA, Campbell O, Heichelheim J. Organizing delivery care: what works for safe motherhood? Bull World Health Organ. 1999:77(5):399-406.

5. Gavin L, Moskosky S, Carter M, Curtis K, Glass E, Godfrey E, Marcell A, Mautone-Smith N, Pazol K, Tepper N, et al. Providing quality family planning services: Recommendations of CDC and the U.S. Office of Population Affairs. MMWR Recommendations Reports. 2014;63(RR-04):1-54.

6. Sustainable Development Knowledge Platform [https:// sustainabledevelopment.un.org/sdg3]. Accessed 26 Apr 2016.

7. Gulliford M, Figueroa-Munoz J, Morgan M, Hughes D, Gibson B, Beech R, Hudson M. What does 'access to health care' mean? J Health Serv Res Policy. 2002;7(3):186-8

8. Ayanore MA, Pavlova M, Groot W. Unmet reproductive health needs among women in some west African countries: a systematic review of outcome measures and determinants. Reprod Health. 2016;13:5.

9. Solar O, Irwin A: A conceptual framework for action on the social determinants of health. Social Determinants of Health Discussion Paper 2 (Policy and Practice). In.; 2010.

10. Darroch JE, Singh S. Trends in contraceptive need and use in developing countries in 2003, 2008, and 2012: an analysis of national surveys. Lancet. 2013;381(9879):1756-62.

11. Overbosch GB, Nsowah-Nuamah NNN, van den Boom GJM, Damnyag L. Determinants of antenatal care use in Ghana. J Afr Econ. 2004:13(2):277-301.

12. Kabir M, lliyasu Z, Abubakar IS, Asani A. Determinants of utilization of antenatal care services in Kumbotso Village, northern Nigeria. Trop Dr. 2005; 35(2):110-1

13. Dong H, Kouyate B, Cairns J, Sauerborn R. Inequality in willingness-to-pay for community-based health insurance. Health Policy. 2005;72(2):149-56.

14. Addai I. Determinants of use of maternal-child health services in rural Ghana. J Biosoc Sci. 2000:32(1):1-15.

15. Babalola S, Fatusi A. Determinants of use of maternal health services in Nigeria--looking beyond individual and household factors. BMC Pregnancy Childb. 2009;9(1):43.

16. Dixon J, Tenkorang EY, Luginaah I. Ghana's National Health Insurance Scheme: a national level investigation of members' perceptions of service provision. BMC Int Health Hum Rights. 2013:13(1):35.

17. Ibiwoye A, Adeleke IA. Does National Health Insurance Promote Access to quality health care? Evidence from Nigeria. Geneva Papers on Risk Insurance - Issues Practice. 2008;33(2):219-33.

18. Odeyemi IA, Nixon J. Assessing equity in health care through the national health insurance schemes of Nigeria and Ghana: a review-based comparative analysis. Int J Equity Health. 2013;12(1):9

19. Ghana Statistical Service, Ghana Health Service, ICF International. Ghana Demographic and Health Survey. Ghana Demographic and Health Survey 2014. Rockville: GSS, GHS, and ICF International; 2014.

20. NPC NPC, ICF II. Nigeria demographic and health survey 2013. Abuja, Nigeria and Rockville, Maryland: NPC and ICF International; 2014

21. Romesburg C: Cluster analysis for researchers: Lulu. com; 2004. 
22. Onah HE, Ikeako LC, lloabachie GC. Factors associated with the use of maternity services in Enugu, southeastern Nigeria. Soc Sci Med. 2006;63(7): 1870-8.

23. Mekonnen $Y$, Mekonnen A. Utilization of maternal health care services in Ethiopia. In: DHS Further Analysis Reports No 38. Calverton, Maryland: ORC Macro; 2002.

24. Ashford L. Unmet need for family planning: recent trends and their implications for programs: population reference bureau; 2003.

25. Ahmed S, Creanga AA, Gillespie DG, Tsui AO. Economic status, education and empowerment: implications for maternal health service utilization in developing countries. PLoS One. 2010;5(6):e11190.

26. Ochako R, Fotso JC, Ikamari L, Khasakhala A. Utilization of maternal health services among young women in Kenya: insights from the Kenya demographic and health survey, 2003. BMC Pregnancy Childb. 2011;11(1):1.

27. Singh K, Osei-Akoto I, Otchere F, Sodzi-Tettey S, Barrington C, Huang C, Fordham C, Speizer I. Ghana's National Health insurance scheme and maternal and child health: a mixed methods study. BMC Health Serv Res. 2015;15:108.

28. Aremu O, Lawoko S, Dalal K. Neighborhood socioeconomic disadvantage, individual wealth status and patterns of delivery care utilization in Nigeria: a multilevel discrete choice analysis. Int J Women's Health. 2011;3:167-74.

29. Asante F, Chikwama C, Daniels A, Armar-Klemesu M. Evaluating the economic outcomes of the policy of fee exemption for maternal delivery care in Ghana. Ghana Med J. 2007:41(3):110-7.

30. Olakunde B. Public health care financing in Nigeria: which way forward? Ann Nigerian Med. 2012;6(1):4.

\section{Publisher's Note}

Springer Nature remains neutral with regard to jurisdictional claims in published maps and institutional affiliations.

Ready to submit your research? Choose BMC and benefit from:

- fast, convenient online submission

- thorough peer review by experienced researchers in your field

- rapid publication on acceptance

- support for research data, including large and complex data types

- gold Open Access which fosters wider collaboration and increased citations

- maximum visibility for your research: over $100 \mathrm{M}$ website views per year

At $\mathrm{BMC}$, research is always in progress.

Learn more biomedcentral.com/submissions 